\title{
Ultrafast x-ray-driven phenomena in nanocrystals: devel- opment and application of powerful simulation tools
}

\author{
Malik Muhammad Abdullah ${ }^{1,2,3, *}$, Zoltan Jurek ${ }^{1,2, * *}$ Sang-Kil Son ${ }^{1,2, * * *}$, and Robin \\ Santra ${ }^{1,2,3, * * * *}$ \\ ${ }^{1}$ Center for Free-Electron Laser Science, Deutsches Elektronen-Synchrotron, Notkestrasse 85, 22607 \\ Hamburg, Germany \\ ${ }^{2}$ The Hamburg Centre for Ultrafast Imaging, Luruper Chaussee 149, 22761 Hamburg, Germany \\ ${ }^{3}$ Department of Physics, University of Hamburg, Jungiusstrasse 9, 20355 Hamburg, Germany
}

\begin{abstract}
We investigate the radiation damage dynamics of nanocrystals at high x-ray intensity, by using time-resolved scattering patterns. We present dynamics simulations for biologically relevant molecules using XMDYN extended to nanocrystals and scattering simulation with XSINC.
\end{abstract}

\section{Introduction}

For imaging nano-sized crystals of biological macromolecules (e.g. proteins) at atomic resolution, one calls for high intensity and short x-ray pulses. The shortcoming of high intensities is the rapid ionization of the atoms on the timescale of a few femtoseconds, which affects the structure of the system. This radiation-induced damage changes the atomic form factors and may induce significant atomic displacement on longer times. Thus, radiation damage eventually changes the scattering pattern. The bottleneck one faces is that it is computationally not feasible to simulate a nanocrystal system with a realistic size using conventional software tools that are capable of following the radiation damage dynamics, required for imaging studies at high $\mathrm{x}$-ray intensity. Here, we present a methodology to overcome this computational bottleneck.

\section{Methodology}

To follow the time-evolution of the nanocrystal, we have developed an extension of XMDYN [1-3], which is a complex simulation tool for modeling dynamics of molecular systems (e.g. biological molecules) irradiated by an intense hard x-ray pulse. The methodology involves the subdivision of a crystal into smaller super-cell units. In order to calculate the dynamics within every unit cell, XMDYN hybrid framework using real space periodic boundary conditions (PBC) is employed. By combining all the units, we simulate the scattering pattern of a crystal larger than the transverse X-ray beam size using XSINC [4], which is a situation commonly encountered in serial femtosecond nanocrystallography experiments

\footnotetext{
*e-mail: muhammad.abdullah.malik@desy.de

**e-mail: zoltan.jurek@desy.de

***e-mail: sangkil.son@desy.de

****e-mail: robin.santra@desy.de
} 
with tightly focused x-ray free-electron laser radiation. Within our approach the formation of the scattering pattern is described by the following equation:

$$
\begin{aligned}
\frac{d I(\mathbf{Q}, \mathcal{F}, \omega)}{d \Omega} & =\mathcal{F} C(\Omega) \int_{-\infty}^{\infty} d t g(t) \sum_{I, r} P_{I, r}(\mathcal{F}, \omega, t) \\
& \times\left|\sum_{\mu} e^{i \mathbf{Q} \cdot \mathbf{R}_{\mu}} \sum_{X} \sum_{j=1}^{N_{X}} f_{X, I_{X, j}^{\mu}}(\mathbf{Q}, \omega) e^{i \mathbf{Q} \cdot r_{X, j}^{\mu}}\right|^{2} .
\end{aligned}
$$

In this equation, $\mathbf{Q}$ is the momentum transfer, $\mathcal{F}$ is the $\mathrm{x}$-ray fluence, which is considered to be homogeneous throughout the crystal, the index $\mu$ runs over all super-cells and $\omega$ is the photon energy. $C(\Omega)$ is a factor depending on the polarization of the x-ray pulse, and $g(t)$ represents the normalized temporal envelope. $f_{X, I_{X, j}^{\mu}}$ is the atomic form factor of the $j$ th atom of species $X$ in the $\mu$ th super-cell, $I_{X, j}^{\mu}$ is the associated electronic configuration, $I=\left\{I_{X, j}^{\mu}\right\}$ denotes a global electronic configuration, $\mathbf{r}_{X, j}^{\mu}$ represents the position vector of the $j$ th atom of species $X$ in the $\mu$ th super-cell, and $r=\left\{\mathbf{r}_{X, j}^{\mu}\right\}$ indicates the set of all atomic positions. $N_{X}$ represents the total number of atoms for species $X$ within a super-cell. $P_{I, r}$ represents the probability of global electronic configuration $I$ and atomic positions $r$ at time $t$, determined by the x-ray induced stochastic dynamics, and $\mathbf{R}_{\mu}$ represents the position of the $\mu$ th super-cell.

\section{Results}

As a demonstration we have considered a molecular species of biological relevance consisting of heavy and light atomic species like $\mathrm{C}, \mathrm{N}, \mathrm{O}$ and $\mathrm{I}$. In the simulations, we consider pulse parameters used for the experiment recently performed at the LCLS free-electron laser [5]. To map out radiation damage as a function of time, we considered an $\mathrm{x}$-ray pump and $\mathrm{x}$ ray probe scheme, varying a time delay between two identical pulses. For both pump and probe pulses the photon energy, was $9.7 \mathrm{keV}$, the pulse duration was $10 \mathrm{fs}$ (FWHM) and the fluence of $1.0 \times 10^{13} \mathrm{ph} / \mu \mathrm{m}^{2}$ (estimated to be at the center of the focus). The beam focus was considered to be $150 \mathrm{~nm} \times 150 \mathrm{~nm}$ in FWHM. Different time delays were considered between $0 \mathrm{fs}$ and $110 \mathrm{fs}$. We calculated the dynamics of the particles within individual crystal unit cells of 184 atoms applying PBC. We repeated the calculations with different random seeds 50 times in order to sample the stochastic dynamics for the numerical evaluation of Eq (1). Figure 1 shows the time evolution of charges. The average charge state goes up to +41 for iodine, which increases further to +47 during the probe pulse. Impact ionization after photoionization and Auger decay plays an important role in stripping off electrons from iodine atoms. Even at the end of the probe pulse, simulations show ionization due to impact ionization for iodine atoms. This shows that highly energetic photo-electrons still exist and can induce more damage at longer time scales. It may be noted that the system after the probe pulse is not yet thermalized.

Fig. 2 represents the scattering intensity for a selected Bragg reflections as a function of time delay. The scattering patterns are obtained using the probe pulse only, whereas the pump pulse is filtered out. One can see a drastic decrease of scattering intensity as a function of time delay in Fig. 2 (a-c). The reason for the decrease in the scattering intensity is the higher depletion of the bound electrons.

\section{References}

[1] Z. Jurek, S.-K. Son, B. Ziaja, and R. Santra, J. Appl. Cryst. 49, 1048-1056 (2016) 

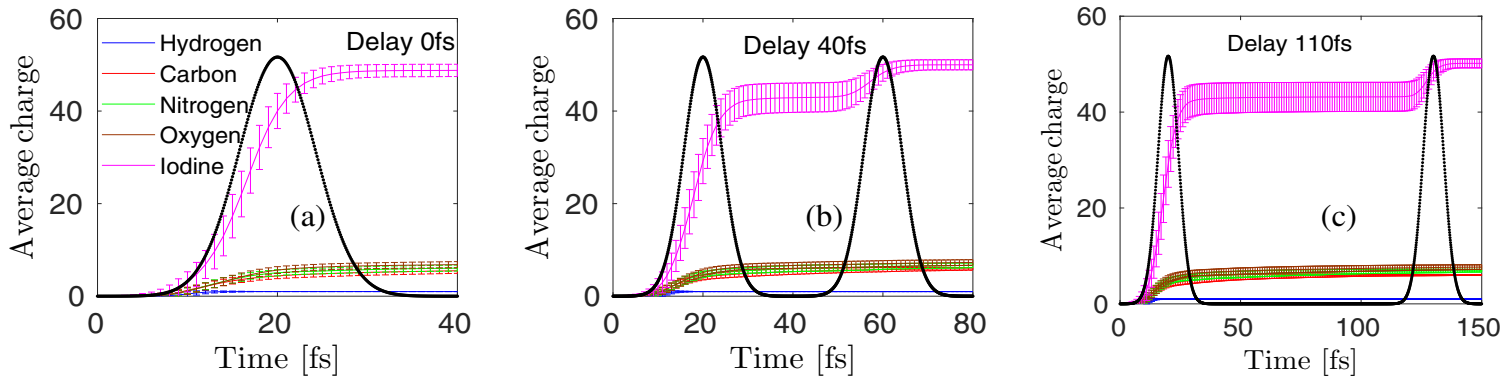

Figure 1. Average charge as a function of time, representing (a) $0 \mathrm{fs}$ delay, where the two pulses are coincident, (b) $80 \mathrm{fs}$ delay and (c) $110 \mathrm{fs}$ delay. The black curve represents the temporal Gaussian envelope of $10 \mathrm{fs}$ FWHM. The fluence considered is $1.0 \times 10^{13} \mathrm{ph} / \mu \mathrm{m}^{2}$, whereas the average charge is calculated using 50 trajectories for each delay.

Bragg reflection (1 111 )

$\log _{10}$ Intensity [arb. units]

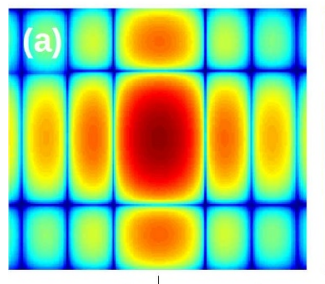

0

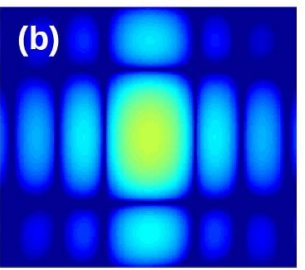

40

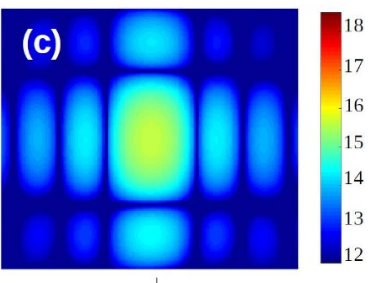

110

Time Delay $[\mathrm{fs}]$

Figure 2. Contour plot for the Bragg reflection $\left(\begin{array}{lll}1 & 1 & 1\end{array}\right)$ in the $\left(\mathrm{Q}_{\mathrm{x}}, \mathrm{Q}_{\mathrm{y}}\right)$ plane in reciprocal space for $1.0 \times 10^{13} \mathrm{ph} / \mu \mathrm{m}^{2}$.

[2] B. Murphy, Z. Jurek, et al., Nat. Commun. 5, 4281 (2014).

[3] T. Tachibana, Z. Jurek, et al., Scientific Reports 5, 10977 (2015).

[4] M. M. Abdullah et al., Struct. Dyn. 3, 054101 (2016).

[5] J. C. H. Spence, A. K. Richard, H. N. Chapman, and D. Oberthuer, "Towards RIP using free-electron laser SFX data," Private Commun. (2016-17). 